\title{
Synthesis, Growth and Characterization of Unidirectional L-Histidine Acetate Single crystal
}

\author{
G. Prabagaran ${ }^{1,2}$, S. Arulmozhi ${ }^{2}$, M. Dinesh Raja ${ }^{2}$ and J. Madhavan ${ }^{2} *$ \\ ${ }^{I}$ Department of Physics, Govt. Arts College (Men), Nandanam, Chennai - 600 035, India \\ ${ }^{2}$ Department of Physics, Loyola College, Chennai - 600 034, India
}

\begin{abstract}
Unidirectional $\langle 100\rangle$ L-Histidine acetate (LHA) single crystal was grown for the first time and reported. The grown crystal was characterized by powder X-ray diffraction (XRD) studies to confirm the crystal structure. The mechanical properties of the grown crystals have been studied using Vickers microhardness tester. The Second Harmonic Generation (SHG) in the sample was confirmed and estimated by Nd: YAG laser employing the Kurtz and Perry powder technique. Thermal stability of the grown crystal was determined by Thermogravimetic (TG) and Differential theromogravimetric (DTG) analysis. Dielectric and photoconductivity studies were also carried out for the grown LHA crystals.
\end{abstract}

Key words: NLO; Unidirectional growth; LHA; UV-Vis-NIR; Thermal studies

\section{Introduction}

Frequency conversion is an important technique for extending the useful wavelength range of lasers. In photonics, a growing need continues for low cost, highly nonlinear, efficient and high quality crystals for optical frequency conversion. Nonlinear optical (NLO) organic materials have received much importance for optical second harmonic generation (SHG) owing to their practical applications in the domain of optoelectronics and photonics. The search for suitable NLO materials exhibiting Second Harmonic Generation (SHG) is the focus of current research. Amino acids are potential candidates for optical Second Harmonic Generation (SHG) because they contain chiral carbon atom and crystallize in noncentro symmetric space groups $[1,2,3]$. Salts of L-histidine have become promising NLO materials after Marcy et al. reported L-histidine tetrafluoborate has higher efficiency than the L-Arginine Phosphate (LAP) [4]. L-histidine acetate dihydrate (LHA) is one such organic nonlinear optical material that belongs to the histidine-complex family. L-histidine Acetate (LHA) has been reported as a promising organic NLO material, which has SHG efficiency three times higher than KDP [5]. In the present study, large size unidirectional LHA single crystal was grown successfully by SR method for the first time. Powder XRD, UV-Vis-NIR, microhardness, thermal and dielectric studies were carried out to characterize the grown crystal. The NLO property of the crystal was confirmed by Kurtz powder technique.

\section{Experimental Procedure}

The compound L-His. $\mathrm{CH}_{3} \mathrm{COOH} .2 \mathrm{H}_{2} \mathrm{O}$ was synthesized by reacting equimolar proportion of L-histidine and acetic acid, in deionised water. The synthesized salt was purified by repeated crystallization. The seed crystal grown by the conventional slow solvent evaporation technique was used for the current study. $<100>$ plane of the seed crystal of LHA was chosen and it was transferred to the saturated solution of LHA in the ampoule.

The experimental setup of SR method consists of growth ampoule made-up of glass with seed mounting pad. The circular shaped heaters were placed at the top and the bottom of the growth ampoule which provides the required experimental temperature for solvent evaporation [6]. The temperature around the growth ampoule is selected based on the solvent used and it is monitored with a temperature controller $\left(40{ }^{\circ} \mathrm{C}\right.$ for top and $34{ }^{\circ} \mathrm{C}$ for bottom).

Growth of highly transparent single crystal of LHA of $10 \mathrm{~mm}$ diameter and $28 \mathrm{~mm}$ length was grown (Fig. 1) in a period of 28 days and reported for the first time. The average growth rate was found to be nearly $1 \mathrm{~mm}$ per day. Thus it is found that the average growth rate of crystal by SR method was higher than the conventional method under prevailing conditions. 


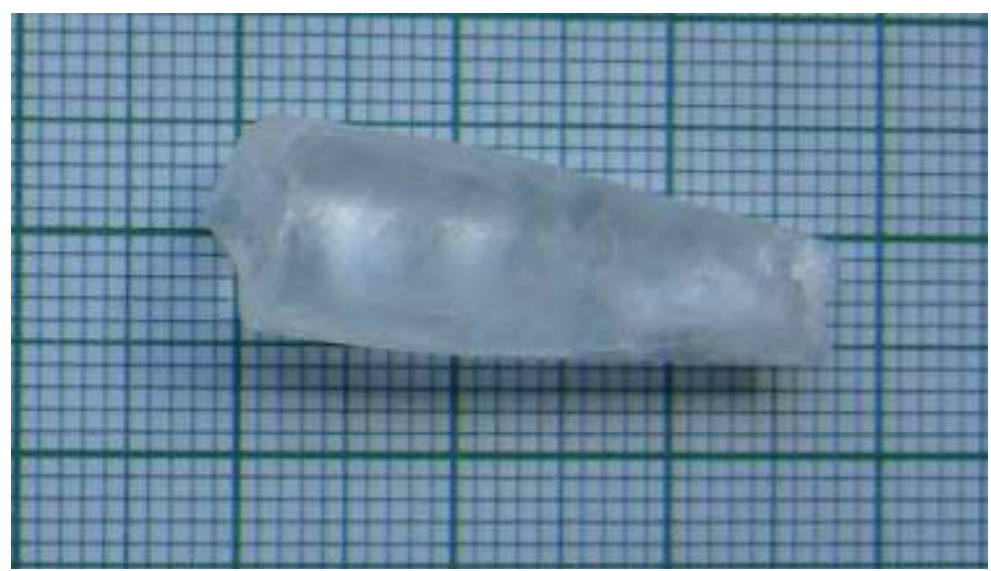

Fig.1. LHA crystal grown by SR method

\subsection{Powder XRD studies}

\section{Results and Discussion}

The structural property of the grown crystal was studied by X-ray powder diffraction technique. Powder X-ray diffraction studies of LHA crystals were carried out, using Siemens D500 X-ray diffractometer

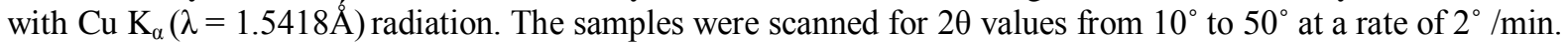
Fig 2 shows the Powder XRD pattern of LHA crystal. The diffraction patterns of LHA crystal was indexed by least square fit method. The lattice parameter values of the pure LHA crystal has been calculated and is well matched with the reported literature. It is seen that the grown crystal crystallizes in triclinic P1 space group and the lattice parameters are shown in Table 1.

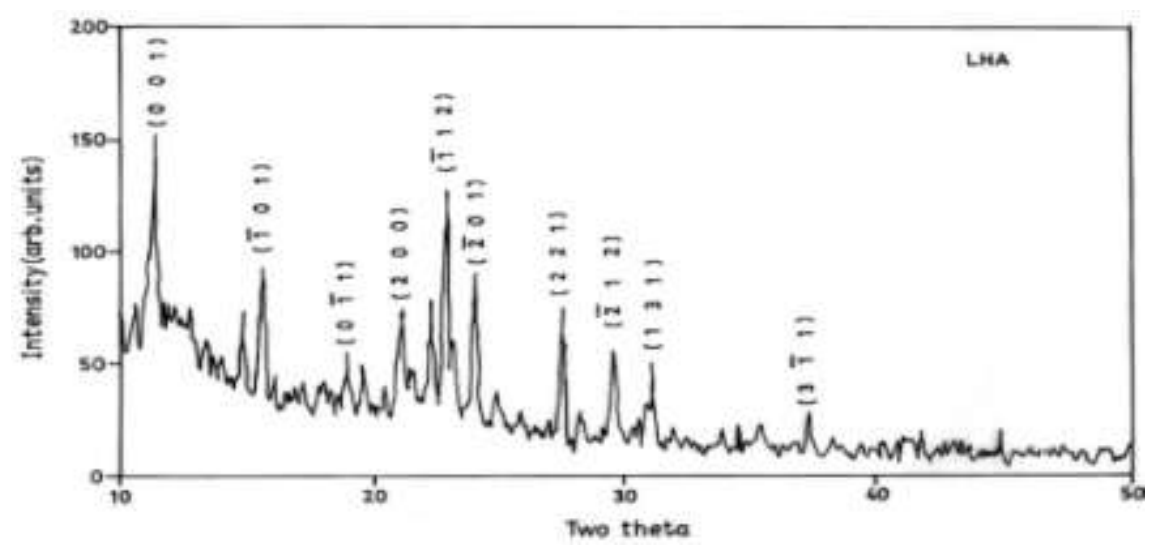

Fig.2. Powder XRD pattern of LHA crystal

Table. 1 Crystal data of LHA crystal

\begin{tabular}{|c|c|}
\hline Empirical formula & $\mathrm{C}_{6} \mathrm{~N}_{3} \mathrm{O}_{2} \mathrm{H}_{10}{ }^{+} \mathrm{CH}_{3} \mathrm{COO}^{-} .2 \mathrm{H}_{2} \mathrm{O}$ \\
\hline Crystal system & Triclinic \\
\hline Space group & P1 \\
\hline $\mathrm{a}(\AA)$ & 8.530 \\
\hline $\mathrm{b}(\AA)$ & 9.150 \\
\hline $\mathrm{c}(\AA)$ & 9.063 \\
\hline$\alpha^{\circ}$ & 61.73 \\
\hline$\beta^{\circ}$ & 86.64 \\
\hline$\gamma^{\circ}$ & 86.28 \\
\hline Volume $\AA^{3}$ & 611.8 \\
\hline
\end{tabular}

\subsection{UV-Vis-NIR spectrum}

The optical absorption plays an important role in identifying the potential of the NLO material. Materials having wide absorption window with reduced absorption around the fundamental and second harmonic wavelength are of greater utility for NLO applications. Optical absorption data were taken on this polished crystal sample of about $4 \mathrm{~mm}$ thickness using a Varian carry $5 \mathrm{E}$ model dual beam spectrophotometer 
between $200-2000 \mathrm{~nm}$. The spectrum (Fig. 3) indicate that the LHA crystal have minimum absorption in the entire visible region. The required properties for NLO activity are minimum absorption and low cut-off wavelength which is evident in the grown crystal.

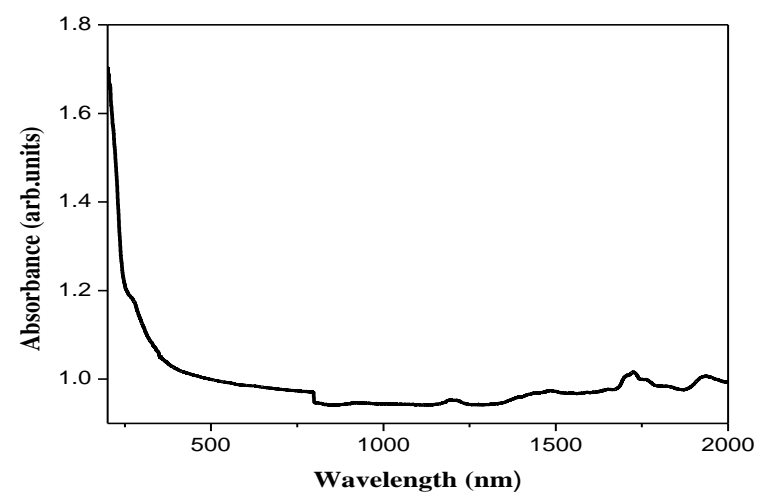

\subsection{NLO studies}

Fig.3. UV-Vis-NIR absorption spectra

Kurtz SHG tests were carried out on the powdered sample of LHA using Nd:YAG Q-switched laser beam as a source [7]. For a laser input of $6.2 \mathrm{~mJ}$, the second harmonic signal $(532 \mathrm{~nm})$ of $91.66 \mathrm{~mW}$ and $352.48 \mathrm{~mW}$ were obtained for KDP and LHA respectively. Thus, the SHG efficiency of LHA is 3.8 times higher than that of KDP.

\subsection{Thermal studies}

The TG and DTG analysis of LHA crystals were done in the nitrogen atmosphere in the temperature range of $28^{\circ} \mathrm{C}-1200^{\circ} \mathrm{C}$ using STA $409 \mathrm{C}$ instrument, at a heating rate of $10^{\circ} \mathrm{C} / \mathrm{min}$. Fig. 4 shows the thermogram of the grown LHA. Due to the loss of lattice water, weight loss of about $35 \%$ is observed for LHA between $115.5^{\circ} \mathrm{C}$ and $274.6^{\circ} \mathrm{C}$. The total weight loss of $59 \%$ is observed between 274.6 and $333^{\circ} \mathrm{C}$ corresponds to the decomposition of pure LHA. $94 \%$ of the sample decomposes at a temperature more than 900 with around $6 \%$ as residue.

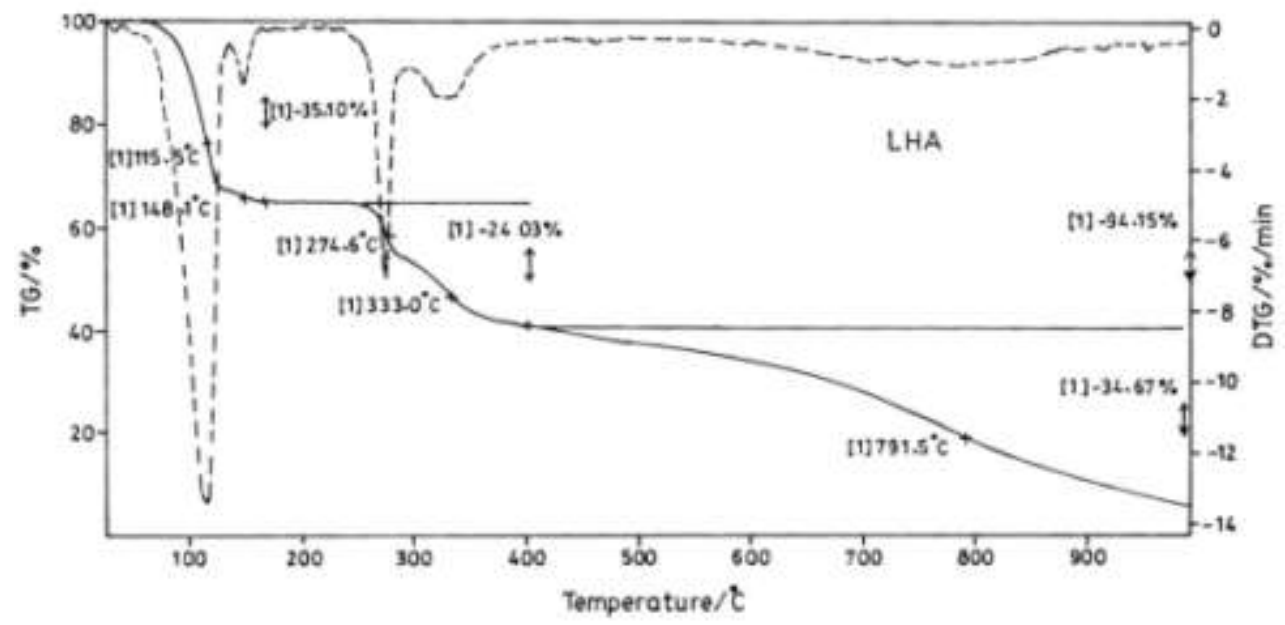

\subsection{Microhardness test}

Fig.4. TGA and DTG thermogram of LHA crystal

Hardness of the material is a measure of resistance, it offers to deformation. Microhardness studies were carried out for the grown crystal using Leitz Wetzlar Vickers microhardness tester by varying the applied load from $5 \mathrm{~g}$ to $50 \mathrm{~g}$. The indentation time was kept as $5 \mathrm{~s}$ for all the loads. Fig. 5 shows the variations of Vickers hardness number with applied load. The value of work hardening coefficient is found to be 1.61 . According to Onitsch, $\mathrm{n}$ lies between 1 and 1.6 for hard materials, and $\mathrm{n}$ is greater than 1.6 for soft materials [8]. Hence, it is concluded that LHA crystal is a soft material. 


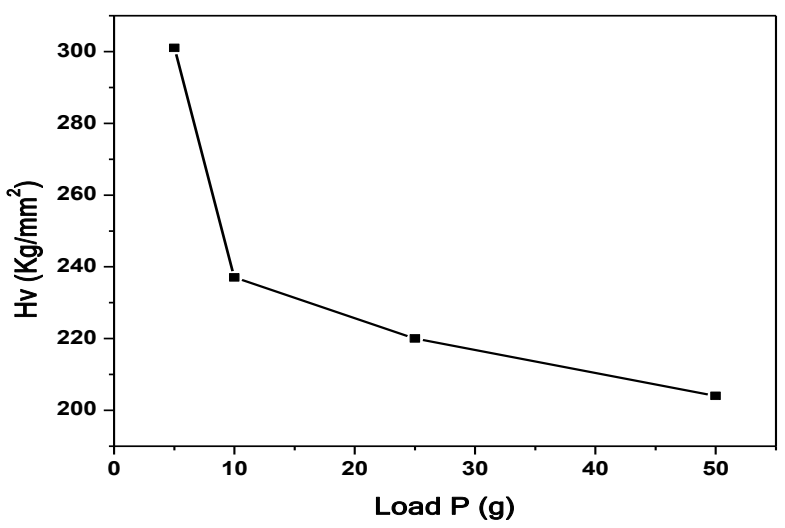

\subsection{Dielectric studies}

Fig 5. Vickers hardness profile

Using the instrument, HIOKI 3532-50 LCR HITESTER, dielectric studies were carried out for the LHA crystal. Fig. 6 shows the variation of dielectric constant with frequency for the LHA crystal. The dielectric constant decreases with applied frequency. The very high value of $\varepsilon_{\mathrm{r}}$ at low frequencies may be due to the presence of all the four polarizations namely; space charge, orientation, electronic and ionic polarization and its low value at higher frequencies may be due to the loss of significance of these polarizations gradually.

The variation of dielectric loss with frequency is shown in Fig. 7. As these materials shows low dielectric loss with high frequency, this sample possesses enhanced optical quality with lesser defects. This is an important parameter of vital importance for NLO materials in their application [8].

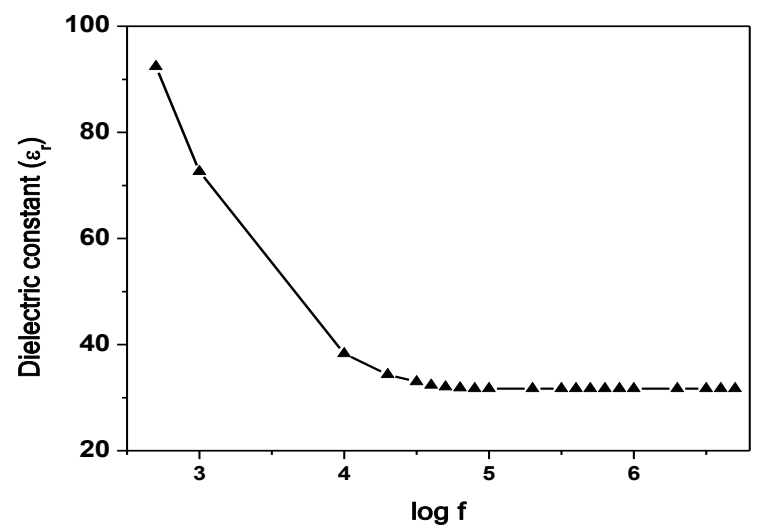

Fig 6. Plot of dielectric constant versus log frequency

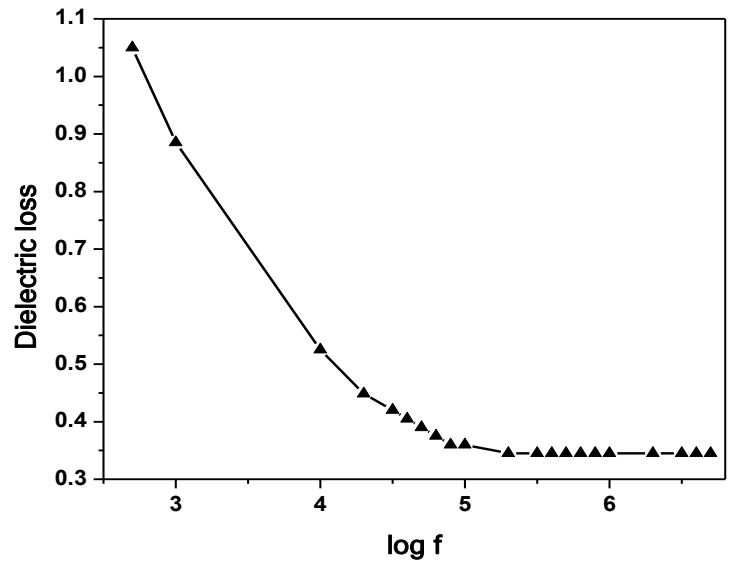

Fig 7. Plot of dielectric loss versus log frequency 


\subsection{Photoconductivity studies}

Photoconductivity studies were carried out for LHA crystal using Keithley 485 picoammeter at room temperature. By connecting the samples to a DC power supply and a picoammeter, dark conductivity of the samples were studied. The light from the halogen lamp (100W) containing iodine vapor is focused on the sample with the help of a convex lens and the photo currents of the sample was measured [9]. The DC inputs were increased from $200 \mathrm{~V}$ to $400 \mathrm{~V}$ in steps and the photo currents were measured for the LHA sample. The variation of both dark current $\left(I_{d}\right)$ and photo current $\left(I_{p}\right)$ with applied field for LHA are shown in Fig. 8. It is seen from the plots that both $I_{d}$ and $I_{p}$ of the sample increase linearly with the applied field. It is also seen that the photo current of LHA crystal is always greater than the dark current and hence LHA exhibit positive photoconductivity. This phenomenon can be attributed to generation of mobile charge carriers caused by the absorption of photons [10].

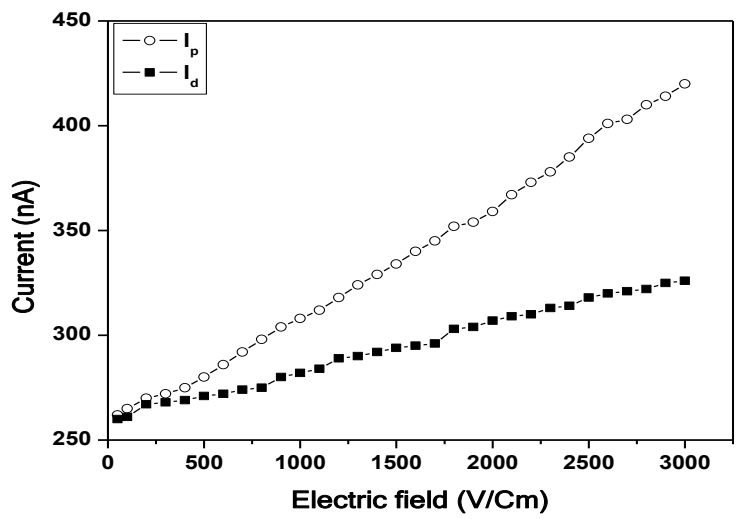

Fig 8. Photoconductivity of LHA

\section{Conclusion}

Good quality unidirectional bulk crystal L-Histidine Acetate (LHA) was grown successfully by SR method. Powder X-ray diffraction studies were carried out, and the lattice parameters were calculated. The LHA crystal is transparent in the entire visible region, and has minimum absorption. The TGA and DTG studies show three stages of weight loss. From the dielectric studies it is seen that the dielectric constant and dielectric loss decreases with frequency. Photoconductivity studies reveal that LHA have positive photoconductivity. Hardness studies show that LHA is a soft material. NLO studies proved that LHA have second harmonic generation efficiency that is 3.8 times that of KDP. The preliminary studies reveal that LHA could be a promising material for nonlinear device fabrication.

\section{References:}

[1]. Senthil. S, Pari. S, Sagayaraj. P, Madhavan. J, Physica B, 2009, 404, 655.

[2]. Senthil S, Pari S, John Xavier R, Madhavan J, Optik., 2012, 123, 104.

[3]. Madhavan. J, Aruna. S, Prabha. K, Packiam Julius. J, Ginson P. Joseph, Selvakumar. S, Sagayaraj. P., Journal of crystal growth, 2006, 293, 409.

[4]. Marcy H.O., Rosker M.J., Warren L.F., Cunningham P.H. and Thomas C.A., Optics Letters, 1995, $20,3,252$.

[5]. J. Madhavan, S. Aruna, A. Anuradha, D. Premanand, I. Vetha Potheher, K. Thamizharasan, P. Sagayaraj, Optical Materials, 2007, 29, 9, 1211.

[6]. Sankaranarayanan K and Ramasamy P, Journal of Crystal Growth; 2006; 292:445.

[7]. Kurtz S K, and Perry T T, J. Appl. Phy., 1968; 39: 3798.

[8]. Ginson P. Joseph, K. Rajarajan, M. Vimalan, S. Selvakumar, S.M. Ravikumar, J. Madhavan and P. Sagayaraj, Materials Research Bulletin 2007, 42, 2040

[9]. K. Rajarajan, G. Mani, I. Vetha Potheher, Joe G.M. Jesudurai, M. Vimalan, Dennis Christy, J. Madhavan and P. Sagayaraj Journal of Physics and Chemistry of Solids, 2007, 68, 2370.

[10]. M. Gulam Mohamed, M. Vimalan, J. G. M. Jesudurai, J. Madhavan, P. Sagayaraj Crystal Research and Technology $2007,42,948$. 